\title{
Unbiased Stratification of Left Ventricles
}

\author{
Rajagopalan Srinivasan, K.S. Shriram, and Srikanth Suryanarayanan \\ Imaging Technologies Lab, GE Global Research, India
}

\begin{abstract}
Image based quantitative stratification of the Left Ventricles (LV) across a population helps in unraveling the structure-function symbiosis of the heart. An unbiased, reference less grouping scheme that automatically determines the number of clusters and a physioanatomically relevant strategy that aligns the intra cluster LV shapes would enable the robust construction of pathology stratified cardiac atlas. This paper achieves this hitherto elusive stratification and alignment by adapting the conventional strategies routinely followed by clinicians. The individual LV shape models $(\mathrm{N}=127)$ are independently oriented to an "attitudinally consistent orientation" that captures the physioanatomic variations of the LV morphology. Affinity propagation technique based on the automatically identified inter-LV_landmark distances is used to group the LV shapes. The proposed algorithm is computationally efficient and, if the inter cluster variations are linked to pathology, could provide a clinically relevant cardiac atlas.
\end{abstract}

\section{Introduction}

The omnipresent manifestation of cardiovascular disease has necessitated the cross-disciplinary convergence towards creating computational tools that enable P4 (personalized, predictive, preemptive and participatory) medicine. Construction of pathology-specific cardiac atlases is a forerunner among these computational tools. By augmenting the epidemiological studies with the associated pathology stratified physioanatomic variations, cardiac atlases can benefit the practitioner, practice and the patient.

Image based quantitation of the pathology associated morphologic alterations and personalization of this knowledge to the patient specific multidimensional data helps in assessing the patient's cardiac wellness. While clinicians have acquired the skills to perform such stratification mentally, automatic stratification requires a machinelearning step that involves collating data across multiple subjects into a statistical atlas that captures the pathology-specific morphology variations.

The atlas construction is typically accomplished through an image alignment process that establishes pair wise correspondence between two datasets and subsequently stitches multiple such correspondences to establish a joint homology. Such approaches suffer from the biased choice of a reference dataset to which the rest are aligned. To overcome this discrepancy, a number of unbiased, simultaneous registration schemes have been proposed [1-3]. These schemes align the multiple subjects to a common coordinate system by determining the most consistent alignment of the 
joint data. Though technically elegant, these techniques do not adhere to the clinician friendly "attitudinally consistent orientation" [4] and require the simultaneous treatment of all the datasets under investigation.

In brain mapping, the Talairach space is often used as the unifying co-ordinate system that provides "attitudinally consistent orientation" to independently map inter and intra- subject brain datasets. Likewise, a unified space could be described to map LVs based on the following facts: (a) the LV pose serves as a unifying reference frame across different subjects [5]; (b) the motion of the LV through the cardiac cycle can be separated into global translation, longitudinal and circumferential shortening or elongation [6]; and (c) acquisition during the diastasic phase of the cardiac cycle provides a dataset with least motion artifacts [7].

By cascading the above-mentioned facts into an algorithmic pipeline, we propose an unbiased, referenceless approach that reorients the shape models of the individual LVs independently into a consistent physioanatomic space, and in the process maximizes the joint similarities between the datasets. The reoriented LV shapes can be subsequently aggregated into a crisp or statistical atlas. However, for a dynamic physioanatomically-varying organ such as the LV, a single crisp exemplar (atlas) cannot account for the complete family of shape space; blind statistical grouping of aligned shapes does not help in understanding the pathological influences. This necessitates the need for LV stratification into tighter subgroups. Though unsupervised, unbiased, and reference less shape clustering techniques exist, lack of methods to estimate the natural number of clusters in a population has probably hindered the construction of unbiased LV groupings. This paper explores the use of affinity propagation [8] to construct unbiased, natural clusters of LV shapes. In combination with the "attitudinally consistent orientation", the proposed stratification strategy helps in constructing physioanatomically-stratified atlases. Linking the physioanatomic variations to the underlying pathology enables the construction of clinically relevant cardiac atlases.

\section{Materials and Methods}

\subsection{Data Acquisition}

The datasets for this study were selected from the cardiac CT scans acquired at Narayana Hrudhayalaya, Bangalore, India using a 64-slice Lightspeed VCT (GE Healthcare) MDCT scanner. Acquisitions from patients with atypical chest pain, those requiring corrective surgery and potentially normal subjects who participated in the mandatory executive screening were included in this study. In accordance with the clinical protocol, the heart rate of the subjects was, where required, ionotropically controlled using beta-blockers. Personalized BMI-adjusted tube current and retrospective ECG gating was used during the acquisition. High temporal resolution images were obtained by reconstructing the data with partial scan reconstruction at $75 \%$ interval (corresponding to the almost no-motion diastasic phase) in R-R ECG cycle.

\subsection{Construction of LV Shape Model}

The LV shape model was built using an interactive segmentation tool available in the GE Healthcare Advantage Windows (AW) workstation. Trained 3D technicians 
sparsely segmented the key LV sections using the border-tracing tool; the sparse segmentations were populated using a shape propagation technique. Out-of-plane edge incoherency was corrected using a topology preserving smoothing method based on curvature flow.

\subsection{Generation of Attitudinally Consistent Orientation}

As mentioned before, the motion of the LV through the cardiac cycle can be distinctly separated into global translation, longitudinal and circumferential shortening or elongation [6]. By independently translating the individual LV shape models to a common origin and reorienting them so that their orthogonal axes correspond to the directions of longitudinal and circumferential scaling, the respective models can be aligned to the clinically recognized "attitudinally consistent orientation". Grouping the LVs in this consistent position facilitates the quantification of the physioanatomic variations in the morphology.

The global translation is synchronized by computing the centroids of the individual LV and shifting them to a common origin. The longitudinal axis of the LV spans across the apex and the base; this axis corresponds to the pose of the LV shape model. The pose is captured from the shape's second order moment via the Principal Component Analysis (PCA). Aligning the dominant orientation of the individual models independently to a "common axis" (in this study $\mathrm{x}$ - axis was used as the common axis) establishes a consistent longitudinal framework across all the models. Figure 1 illustrates the longitudinal alignment.
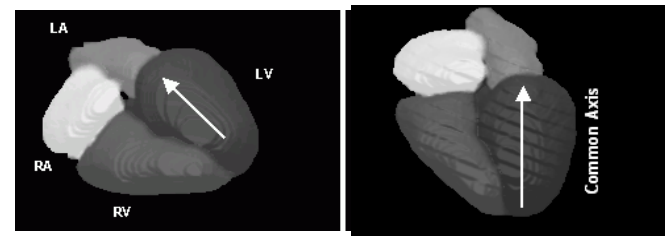

Fig. 1. Consistent inter-subject LV pose (right) is achieved by independently aligning the principal Eigen vectors of the individual LV shape models (left) to a common axis

While the PCA based alignment provides a referenceless, unbiased longitudinal orientation consistency across the LV shape models, it does not guarantee a consistent in-plane orientation. Correcting for this inconsistency in an independent, unbiased and referenceless manner is a challenge and requires exploiting the salient features of the cardiac architecture.

Within the LV, an organ in perpetual flux, the aortic vestibule and the immediate vicinity of the mitral valve are stationary across the cardiac cycle [5]. Identifying and suitably orienting key landmarks within this region provides a consistent in-plane orientation and hence better overall alignment. These landmarks were identified by traversing through the medial surface (MS) of the LV shape model.

A number of efficient algorithms exist for constructing the MS of a given shape. In this study, the Parameter controlled skeletonization proposed in [9] was used. Briefly, the method uses the fact that any point on the MS should be the local maxima in the 

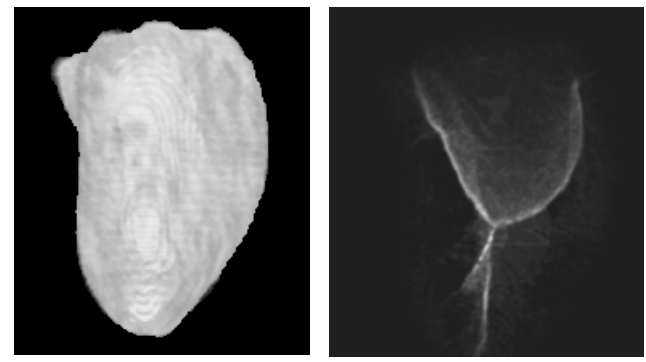

Fig. 2. Parameter-skeleton based MS (right) of a LV shape model (left)

distance transform space. The local maxima locations greater than the mean neighborhood distance is identified as a candidate location in MS. Figure 2 shows the MS generated by this method for a representative LV.

As clearly seen in Figure 2, the MS of LV originates at the apex and bifurcates towards the aortic and mitral valves. The landmarks of our choice lie at the terminal of the "Y". The landmark corresponding to the aortic valve is used to fix the in-plane rotation. Since the aortic valve is closed during the diastasic phase, the distance coding of the prong terminating on this valve will be stronger (this can be clearly seen in the left prong of Figure 2). The plane formed by the principal eigen vector of the LV and the aortic valve (terminal of the dominant prong of the MS) is rotated about the "common axis" until it aligns with the XZ plane as shown in Figure 3. This procedure ensures that all the datasets are in an "attitudinally consistent orientation" that captures the physioanatomic variations of the LV morphology.

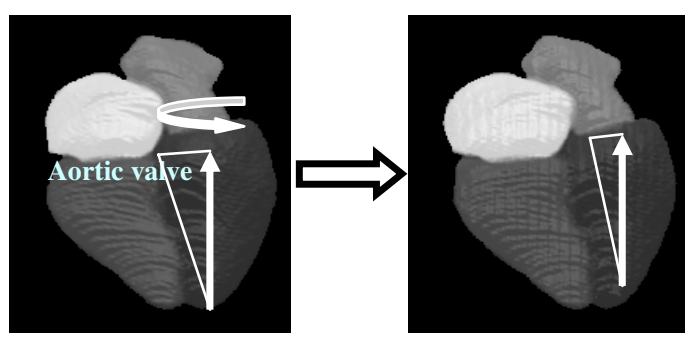

Fig. 3. In-plane alignment of using planed formed by LV principal axis and the aortic valve

\subsection{Stratification of LV Shapes}

As mentioned before, crisp or statistical aggregation of consistently oriented LV models does not provide a clinically relevant cardiac atlas. An unsupervised clustering technique that automatically finds the natural number of clusters is required to create an unbiased stratification of LVs. Affinity propagation [8] readily meets this stringent requirement. Briefly, affinity propagation iteratively finds clusters given pair wise 
similarities of a point cloud of n-dimensional data. Further to resolving the clusters in the point cloud, the algorithm identifies the exemplar that is most 'central' to each of the clusters.

In each iteration, every candidate $i$ is considered as a potential exemplar of a cluster and its 'responsibility' to every potential member $k$ of the cluster computed as:

$$
r(i, k)=s(i, k)-\max _{k^{\prime} \neq k}\left\{a\left(i, k^{\prime}\right)+s\left(i, k^{\prime}\right)\right\}
$$

where, $s(i, k)$ is the similarity between candidates $i$ and $k$ and the 'availability' $a(i, k)$ of data point $k$ to a cluster exemplified by $i$ is defined as:

$$
a(i, k)=\min \left\{0, r(k, k)+\sum_{i \notin\{i, k\}} \max \left\{0, r\left(i^{\prime}, k\right)\right\}\right\}
$$

Iteratively, competing exemplars claim data points at every turn and convergence is achieved when data points coalesce into distinct clusters.

For clustering the LV shapes, similarity metric based on the inter-distances between the apex(Ap), aortic valve (Av) and mitral valve (Mv) as deduced from the terminals of the dominant "Y" in the medial surface was used. Specifically, the similarity metric $s(i, k)$ between datasets $i$ and $k$ is defined as

$s(i, k)=\left(\left(\left(D\left(A p_{i}, A v_{i}\right)+D\left(A p_{i}, M v_{i}\right)+D\left(A v_{i}, M v_{i}\right)\right)-\left(\left(D\left(A p_{k}, A v_{k}\right)+D\left(A p_{k}, M v_{k}\right)+D\left(A v_{k}, M v_{k}\right)\right)\right)^{2}\right.\right.$ where $D(x, y)$ is the distance between points $x$ and $y$.

\section{Results}

On a database of 127 LV shape models, the affinity propagation yielded six unique clusters based on the similarity measure described above. The glyphs in Figure 4 shows the pair wise similarities before and after stratification in the form of a "confusion' matrix; the darker shade implies a low similarity between two datasets. Results for the LV landmark based stratification (right panel) qualitatively reveals the maximization and minimization respectively of intra (diagonal sub blocks) and inter (offdiagonal sub blocks) cluster similarities.

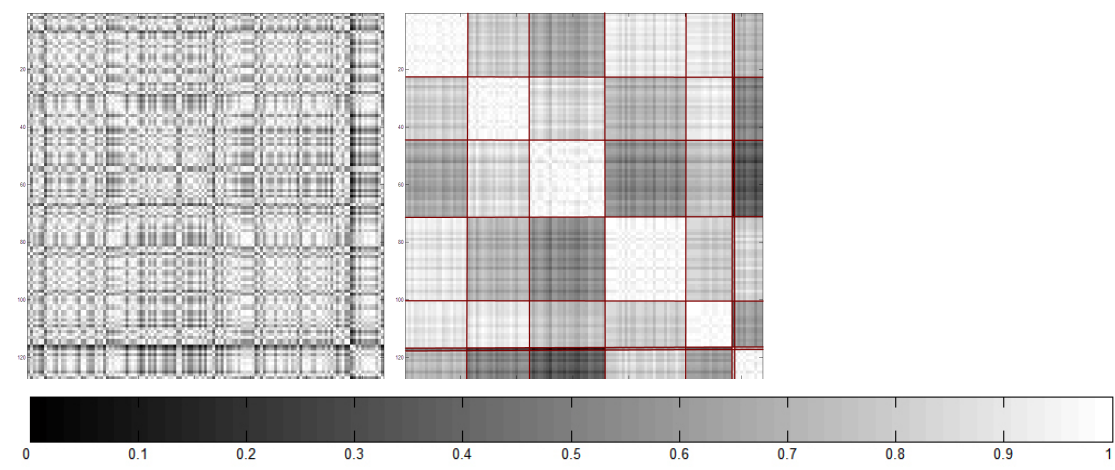

Fig. 4. Pair wise similarity between LV shape models before (left) and after (right) affinity propagation based clustering. Lighter the shade, higher the similarity. 

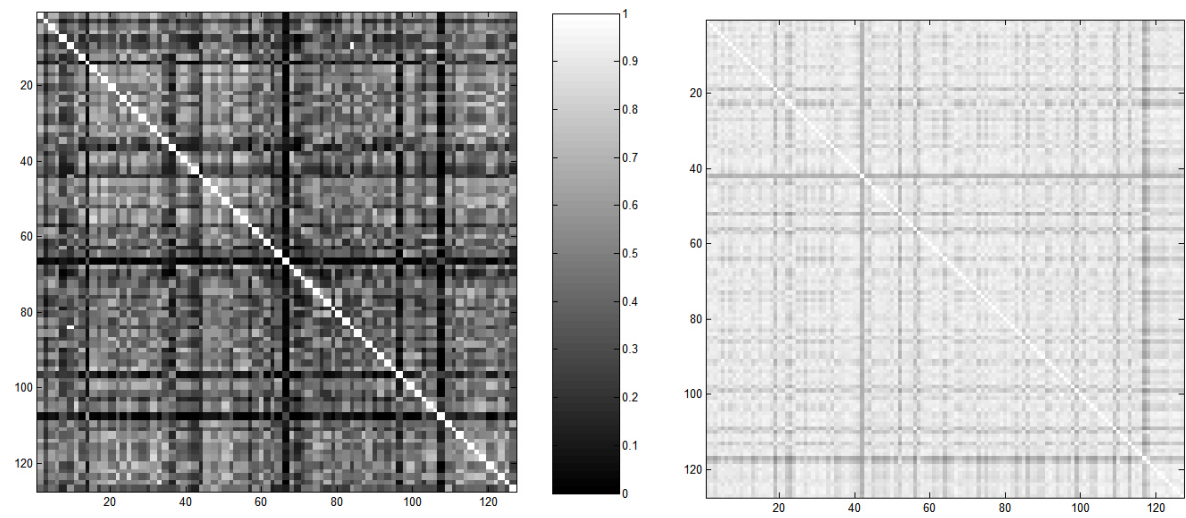

Fig. 5. Pair wise DSCs between the LV shapes before (left) and after (right) independent orientation of the individual LVs into the "attitudinally consistent orientation" space

Quantitative efficacy of the stratification was established by computing the Analysis of Similarity (ANOSIM) R that indicates the magnitude of difference among clusters. Value of 1 indicates that the communities completely differ among defined
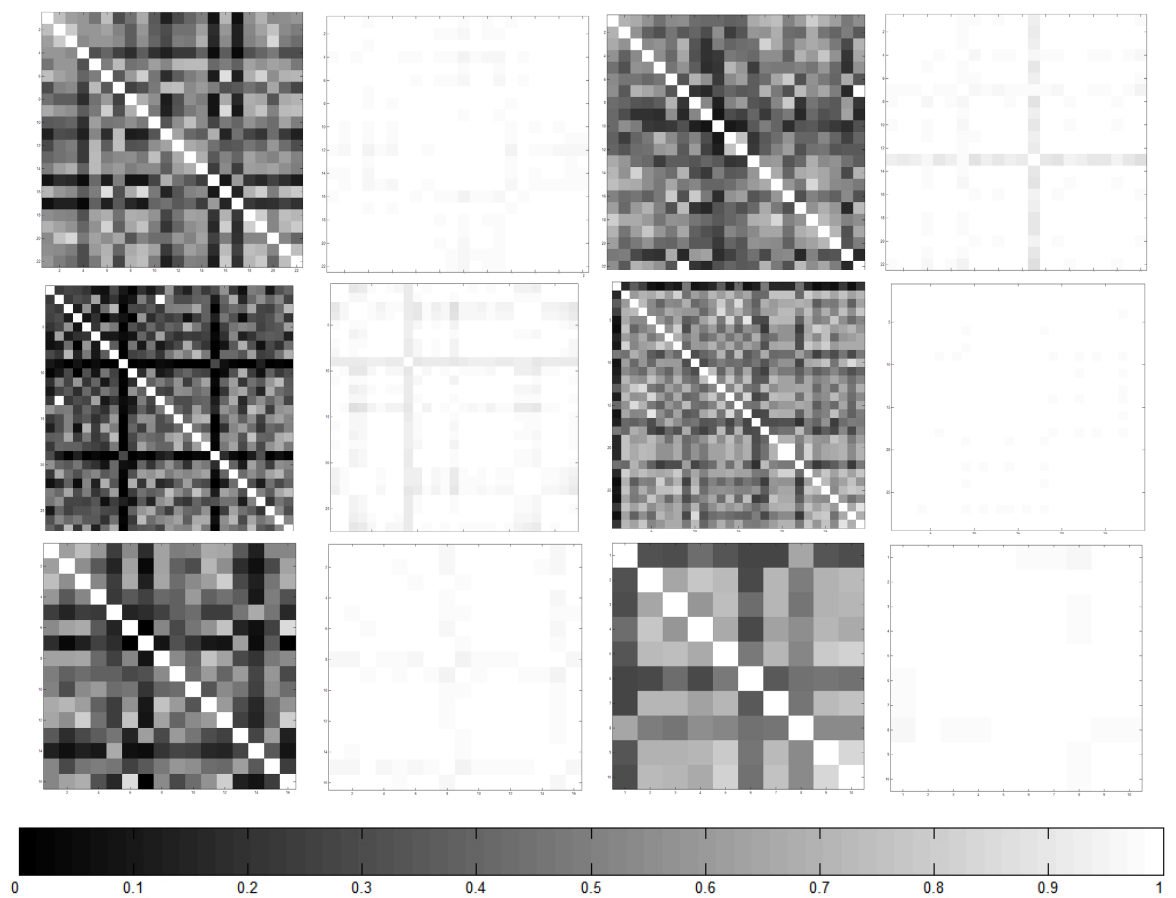

Fig. 6. Pair wise DSC between LV shapes within the six clusters. The DSCs shown in the left and right columns correspond respectively to those before and after the independent alignment to the "attitudinally consistent orientation" space. 


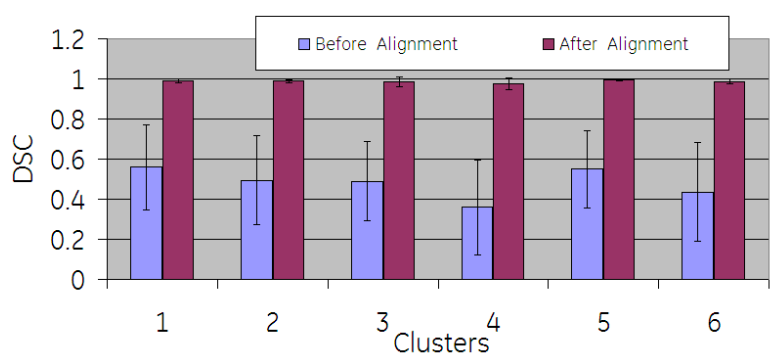

Fig. 7. Pair wise DSC of LV shape volumes in each of the six clusters before and after alignment. The mean DSC shows marked improvement after alignment; concomitantly, the standard deviation has also reduced in the aligned space.

groups, value of 0 indicates no difference among groups. The combined $\mathrm{R}$ for the six LV clusters was found to be $0.9213 \pm 0.023$ highlighting the greater agreement of candidates within each of the clusters.

To capture the efficacy of the "attitudinally consistent orientation" step, pairwise similarity between the models was computed both in the native and aligned space. Dice Similarity Coefficient (DSC) was used as the similarity metric. The glyphs in Figure 5 show the effect of the independent alignment of the individual LVs to the "attitudinally consistent orientation" space. It is clear that the DSC numbers show marked improvement after the unbiased, reference less, anatomically relevant alignment indicating closeness of match. The results also attest the fact that much of the mismatch across LVs can be accounted for by an affine transformation.

Figure 6 shows the pair wise DSC for the LVs within the six clusters. The graph in Figure 7 shows the mean DSCs before and after alignment of the LVs within the six clusters; the statistics agrees with the visual glyphs shown in Figure 7.

\section{Discussion and Conclusions}

The quintessential feature for grouping LV across multiple subjects should preferably be one that has a coeval existence across the life span of the humans. The ballooning of the secondary myocardium from the outer curvature of the "Y" shaped primary heart tube results in the formation of the atrial and ventricular chambers [10]. The apical portion of the left ventricle (LV) originates from the stem of the primary heart tube. Subsequently the mitral and aortic valves develop along the prongs of the heart tube. The signature of the $\mathrm{Y}$ tube about which the LV twirled around can be clearly seen even in adult hearts from its medial axis (Figure 2). This justifies the clinical relevance of the mitral-aortic-LV_base landmarks as key points for LV stratification.

Grouping data automatically into natural clusters has hitherto been a hard problem. The advent of affinity propagation has enabled the possibility of creating unbiased clusters. By combining the quintessential LV landmarks with a robust affinity propagation based LV stratification and unbiased, referenceless, independent orientation of the LVs into "attitudinally consistent orientation" space, this paper made the first attempt to represent multi subject Left Ventricles within a unified space to optimally characterize the physioanatomic variations. By linking the physioanatomic variations 
to the underlying pathology clinically relevant cardiac atlases can be created. While the computational complexity is not addressed here, it should be convincing that given the simplicity of individual steps the turn-around time for stratification is orders of magnitude faster than existing techniques that simultaneously operate on all the datasets. The proposed stratification approach opens up the possibility of image based personalized, preemptive, predictive and participatory medicine.

\section{References}

1. Bhatia, K.K., et al.: Consistent group-wise non-rigid registration for atlas construction. In: Proc. IEEE Symposium on Biomedical Imaging (ISBI), pp. 908-911 (2004)

2. De Craene, M., et al.: Multi-subject registration for unbiased Statistical Atlas Construction. In: Barillot, C., Haynor, D.R., Hellier, P. (eds.) MICCAI 2004, Part I. LNCS, vol. 3216, pp. 655-662. Springer, Heidelberg (2004)

3. Zollie, L., et al.: Efficient population registration of 3D data. In: Liu, Y., Jiang, T., Zhang, C. (eds.) CVBIA 2005. LNCS, vol. 3765, pp. 291-301. Springer, Heidelberg (2005)

4. McAlpine, W.A.: Heart and Coronary Arteries. An anatomical atlas for Clinical Diagnosis, Radiological Investigation, and Surgical Treatment. Springer, Berlin (1995)

5. Edwards, L.: The Vortex of Life. Floris Books (1993)

6. Gould, K.L., et al.: Analysis of wall dynamics and directional components of left ventricular contraction in man. Am. J. Cardiol. 38, 322 (1976)

7. Mahesh, M., Cody, D.D.: Physics of Cardiac imaging with multiple-row detector CT. Radiographics 27, 1495-1509 (2007)

8. Frey, B., Dueck, D.: Clustering by Passing Messages between Data Points. Science 315, $972(2007)$

9. Gagvani, N., Silver, D.: Parameter controlled skeletonization of three dimensional objects. Technical Report CAIP-TR-216, Rutgers State University of New Jersey (1997)

10. Moorman, A., et al.: Development of the heart: formation of the cardiac chambers and arterial trunks. Heart 89, 806-814 (2003) 